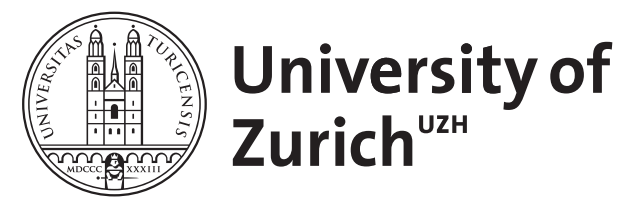

\title{
Rehabilitation and plasticity
}

Luft, A R

\begin{abstract}
Therapies for effective neurorehabiltiation are in part based on brain mechanism commonly described as neuroplasticity. These therapeutic approaches emphasize the re-learning of functionality that was lost due to the injury through reorganization of neural circuits in the remaining intact tissue. Important elements of these therapies are intensive and repetitive training, motivation and potentially interactive devices (therapy "robots") and supportive therapies such as brain stimulation or plasticity inducing medications. Because neuroplasticity-based interventions are complex and multifactorial optimized treatment protocols have to be developed before large clinical trials can provide the evidence of efficacy.
\end{abstract}

DOI: https://doi.org/10.1159/000348879

Posted at the Zurich Open Repository and Archive, University of Zurich

ZORA URL: https://doi.org/10.5167/uzh-84739

Journal Article

Accepted Version

Originally published at:

Luft, A R (2013). Rehabilitation and plasticity. Clinical Recovery from CNS Damage. Frontiers of Neurology and Neuroscience, 32:88-94.

DOI: https://doi.org/10.1159/000348879 


\section{Chapter: Rehabilitation and Plasticity}

Andreas R. Luft

Neurology, University Hospital of Zurich, Switzerland

\section{Introduction}

Neurorehabilitation is a quickly developing field accelerated by advances in neurosciences and rehabilitation engineering. In contrast to acute care medicine with its goal to cure, neurorehabilitation aims at minimizing disability and accelerating the return to an independent life. Naturally, it has to focus on all the deficits and dysfunctions of a patient that stand against these goals. Typically, physical (movement), communicational and cognitive deficits occur and interfere with each other. Therefore the classical neurorehabilitative approach combines physio- and occupational therapy with speech and language and cognitive training, the latter typically provided by neuropsychologists.

Training, i.e. repetitive engagement in challenging exercises is still the mainstay of neurorehabilitative treatment protocols. Training shares certain neural mechanisms with healthy learning, i.e., what is commonly described as neuroplasticity, although it is unclear in how far these processes overlap or share common mechanisms.

Whether training can be effectively enhanced by plasticity-encouraging interventions such as brain stimulation or certain drugs, remains to be proven by clinical research.

What is known from experience as well as science is that higher intensity is more effective than lower intensity (Kwakkel et al. 2004, Teasell et al. 2005), although this may not hold true for early time points after injury (in this case, stroke, Dromerick et al. 2009). High intensity training requires excellent patient compliance and motivation, the major limiting factors for successful training.

\section{Mechanisms of training}

\section{Animal models}

Like motor learning in healthy, rehabilitative training after a lesion to motor cortex leads to modifications in motor cortices adjacent to the lesion. Motor representations change in size and shape (Nudo and Milliken. 1996,Nudo et al. 1996) and new connections form within and between sensory and motor cortical networks (Dancause et al. 2005). The representations of complex movement patterns that can be evoked by long highfrequency pulses to the injured hemisphere - a possible reflection of a memory trace for complex movements - are lost after a cortical lesion. With rehabilitative training they recover; the degree of restoration of complex movement patterns evoked by brain stimulation correlates with behavioral recovery (Ramanathan et al. 2006).

Plasticity also occurs in the uninjured hemisphere contralateral to the lesion. Biernaski and Corbett reported enlarged dendritic trees in the uninjured hemisphere in animals that were trained in an enriched environment after a focal lesion to motor cortex as compared with control animals that received standard therapy (Biernaskie and Corbett. 2001). Indirect evidence for plastic modification was also found in other brain areas that are in involved in motor control. In the cerebellum of rats that were subjected to repeated treadmill training after a middle cerebral artery occlusion, the $25-\mathrm{kDa}$ synaptosomal-associated protein and glial fibrillary acidic protein - markers of synaptic plasticity - were up-regulated as compared with untrained controls (Mizutani et al. 2010). Similarly in thalamus, synaptic plasticity indexed by synaptophysin expression was increased in the damaged hemisphere in animals that were trained on a rotarod (skilled locomotor training) as compared to a treadmill (locomotor training of lower difficulty) or no training (Ding et al. 2003). In the striatum, expression levels of glutamate, brain-derived neurotrophic factor (BDNF), and p-synapsin I were increased by treadmill training after middle cerebral artery occlusion (Chang et al. 2009). However, the type motor training certainly plays a role in the pattern of plastic modification across the rat brain: Whereas forelimb precision movement training (typically single pellet reaching tasks) depends more on cortical plasticity, locomotor training likely induces reorganization in larger motor control networks including cerebellum, basal ganglia and thalamus.

\section{Studies in humans}

In humans, evidence for plastic modification of neural circuits comes from imaging and electrophysiology. Task-related activation in motor cortices or, in case of cortical lesions, the periinfarct cortex is modified 
during the course of recovery. After corticospinal tract infarction, initial overactivation of the contralesional motor cortex (ipsilateral to the moving hand) reverts to ipsilesional (contralateral) dominance as recovery progresses (Kozlowski et al. 1996,Ward et al. 2003). The presence of motor potentials evoked from the lesioned hemisphere using transcranial magnetic stimulation (TMS) and corticospinal tract integrity in diffusion tensor magnetic resonance imaging predicted the response to arm training in chronic stroke survivors (Stinear et al. 2007). Interfering with the contralesional hemisphere by transcranial magnetic stimulation (TMS) did not affect reaction times with the paretic hand in stroke survivors with varying degrees of recovery at least 2 years after stroke; but, interfering with the ipsilesional motor cortex did (Werhahn et al. 2003). This emphasizes the role of the ipsilesional hemisphere (contralateral to the moving limb) for successful recovery.

In predicting therapy gains, the degree of injury to descending tracts from primary motor and premotor cortices is an important surrogate marker. It correlated with gains achieved after hand robotic therapy (Riley et al. 2011). Bilateral arm training improved the arm impairment in chronic stroke survivors. This improvement was paralleled by a increase in activation of bilateral premotor cortices after as compared to before training (Luft et al. 2004). As compared with conventional physiotherapy according to neurodevelopmental principles, bilateral arm training was as effective but showed a different neural response: where conventional physical therapy had no effect on brain activation, bilateral training lead to an increase in bilateral premotor cortex activation (Whitall et al. 2011). This indicated that different therapies operate through different mechanisms even if they induce a comparable behavioral response.

\section{Therapeutic principles}

\section{Timing}

When to start rehabilitation after stroke or trauma is a matter of debate. It seems that forcing an animal to train too early increases the infarct volume (Kozlowski et al. 1996). On the other hand, stroke induces the expression of specific pro-plastic genes defining an optimal time period in which training should be most effective (Carmichael et al. 2005).

In humans, using high-intensity constraint induced movement therapy (CIMT, 3 hours of training based on shaping principles plus immobilizing the intact limb for $90 \%$ of the waking hours for 10 days) starting around 10 days after stroke results in adverse outcomes as compared with standard occupational therapy and standard CIMT (two hours of shaping plus 6 hours of immobilization per day Dromerick et al. 2009). In contrast, Bernhardt and coworkers suggested that early - within 24 hours - mobilization, i.e., getting the patient out of bed, improves the outcome three months after the stroke (Bernhardt et al. 2008). This finding awaits confirmation in a larger sample.

\section{Intensity}

Many studies have compared different training therapies but have shown similar therapeutic responses. Experience suggests that the intensity of training is a major determinant of the training effect. Additional leg training resulted in better walking ability and arm training in better dexterity as compared with immobilization in subacute stroke survivors enrolled within 14 days of stroke onset indicating that the intensity of training counts (Kwakkel et al. 1999). Similarly, a community-based physical activity program improved walking velocity, balance and quality of life measures as compared with standard care also emphasizing training intensity (Stuart et al. 2009). On the other hand as mentioned above, high intensity early after stroke may result in adverse functional outcomeds (Dromerick et al. 2009).

\section{Motivation}

The main obstacles that prevent high intensity training are fatigue and motivation. Fatigue can be counteracted by sufficient interruptions and rest periods. Motivation requires a motivating environment, positive feedback from the therapist or a training device that specifically addresses motivational aspects, e.g., by providing rewarding schemes in a gaming environment. How to optimally use motivational therapy to improve training outcomes is still unclear.

\section{Elements of effective training}

Little is known about which training principles are most effective and for whom. Active training is better than passive movement, but also mental imagery of movement has a training effect in combination with active training (Barclay-Goddard et al. 2011). Bilateral training is similarly effective as unilateral training 
when looking at the population mean; individual patients may respond better to one or the other (Whitall et al. 2011). It remains unknown which factors predispose a patient to respond to a specific treatment.

One of the largest randomized controlled trials ( $n=222$ participants) in neurorehabilitation showed that constraint induced movement therapy (CIMT) is superior to conventional care in patients between 3 and 9 months after a stroke (Wolf et al. 2006). CIMT was developed on the basis of the finding that disuse of an extremity leads to functional deterioration. The constraint element enforces active movement of the affected limb by immobilizing the unaffected. While this trial clearly demonstrated the lasting benefits of CIMT, the controlled comparison depends on the nature of conventional care. In most countries and medical systems conventional care in the time frame of 3 to 9 months is limited to low frequency outpatient therapy, e.g. physiotherapy and occupational therapy once a week. It seems expected that such low intensity is less effective than CIMT which engages patients in training for several hours per day. Hence, it is unknown whether it is simply the intensity that renders CIMT more effective, or it is the strictly unilateral nature of the training, or any other element of therapy.

Progression is another therapeutic principle that may be a key to success. Adding to the complexity, the loading or the velocity of training provides a constant stimulus for learning as the patient continues to improve (Ellis et al. 2009).

\section{Therapy using robots}

Robots were initially developed as assistants to the therapist enabling highly repetitive, uniform passive movements. In patients with spinal cord injury, robotic gait training may be superior to standard overground training (Alcobendas-Maestro et al. 2012) but further studies are needed to confirm these differences. For patients with brain lesions, various studies using different upper or lower extremity robotic training devices were performed and showed inconsistent results ranging from inferiority to equality as compared with conventional physiotherapy (for example see Lo et al. 2010,Hidler et al. 2009).

Robots, however, enable a form of standardized precision training that differs from training under the direction or with the assistance of a therapist. Robots may complement therapist-based training. Precision movement training is often implemented in a computer game. Gaming environments may also increase the motivation for training by introducing reward or competition with others. Finally, robots may partially support the patient's movement (assistance mode) or may perturb the patient's movement, e.g., by applying forces that counteract the patient. Whether assistance or perturbation per se are effective training elements remains to be investigated.

\section{Supportive therapies}

If training is performed in a setting in which plastic reorganization of the brain is facilitated, its effects may be higher, faster and/or longer lasting. Repetitive transcranial magnetic stimulation (rTMS) or transcranial direct current stimulation (tDCS) provide such a setting. They likely work by modulating cortical excitability (in the case of rTMS by increasing or decreasing excitability depending on the frequency of the repetitive stimulation, Corti et al. 2012). As compared with SHAM stimulation, 10 sessions of rTMS as an adjunct to conventional physical and occupational therapy starting 5-10 days after stroke improved disability (as measured by the Barthel index) and impairment (NIHSS, Khedr et al. 2005). In a small randomized trial, rTMS in combination with task-oriented walking exercises improved gait symmetry more than walking exercises alone (combined with SHAM stimulation, Wang et al. 2012). tDCS in combination with conventional physical therapy improved arm impairment and ADL function more than physical therapy alone (combined with SHAM stimulation, Wu et al. 2012). Positive effects of rTMS and tDCS were also reported for the treatment of aphasia (Monti et al. 2008, You et al. 2011).

Drugs potentially improve the effects of rehabilitative training interventions. One larger trial in 118 acute ischemic stroke patients found that adding fluoxetine to standard rehabilitation improves motor impairment at three months (Chollet et al. 2011). The mechanisms by which this selective serotonin reuptake inhibitor exerts this effect are unknown.

\section{Evidence in neurorehabilitation}

Neurorehabilitative interventions are multidimensional often combining different treatments such as exercise and brain stimulation. Finding optimal therapy protocols is much more complex than in pharmacological therapy because many parameters need to be optimized: timing, intensity, duration, rest periods etc. The design of a clinical trial in neurorehabilitation is further complicated by the fact that valid control interventions or groups are difficult to define. Because there is no placebo pill to a training therapy, it is 
difficult and often impossible to double/single-blind the trial. This leaves the investigation vulnerable to placebo effects, e.g., seeing an expensive robot equipment may introduce a placebo effect over the conventional physical therapist. In addition, the outcome measures and scales for movement, speech language and cognitive function all have their shortages. There is little agreement which scale best reflects a clinically relevant treatment effect. Because training interventions require large amounts of therapist time, trials in neurorehabilitation are expensive.

These factors all contribute to the fact that little evidence exists in neurorehabilitation today. Large and expensive trials are risky as long as optimal treatment protocols are not known. Small studies with less than 100 participants are common. Clinical guidelines therefore remain brief and leave much room for personal experience as well as treatments that can still be considered experimental.

\section{References}

Alcobendas-Maestro M, Esclarín-Ruz A, Casado-López RM, Muñoz-González A, Pérez-Mateos G, González-Valdizán E, Martín JL. Lokomat Robotic-Assisted Versus Overground Training Within 3 to 6 Months of Incomplete Spinal Cord Lesion: Randomized Controlled Trial. Neurorehabil Neural Repair. 2012. Barclay-Goddard RE, Stevenson TJ, Poluha W, Thalman L. Mental practice for treating upper extremity deficits in individuals with hemiparesis after stroke. Cochrane Database Syst Rev. 2011;5:CD005950.

Bernhardt J, Dewey H, Thrift A, Collier J, Donnan G. A very early rehabilitation trial for stroke (AVERT): phase II safety and feasibility. Stroke. 2008;39:390-396.

Biernaskie J, Corbett D. Enriched rehabilitative training promotes improved forelimb motor function and enhanced dendritic growth after focal ischemic injury. J Neurosci. 2001;21:5272-5280.

Carmichael ST, Archibeque I, Luke L, Nolan T, Momiy J, Li S. Growth-associated gene expression after stroke: evidence for a growth-promoting region in peri-infarct cortex. Exp Neurol. 2005;193:291-311.

Chang HC, Yang YR, Wang SG, Wang RY. Effects of treadmill training on motor performance and extracellular glutamate level in striatum in rats with or without transient middle cerebral artery occlusion. Behav Brain Res. 2009;205:450-455.

Chollet F, Tardy J, Albucher JF, Thalamas C, Berard E, Lamy C, Bejot Y, Deltour S, Jaillard A, Niclot P, Guillon B, Moulin T, Marque P, Pariente J, Arnaud C, Loubinoux I. Fluoxetine for motor recovery after acute ischaemic stroke (FLAME): a randomised placebo-controlled trial. Lancet Neurol. 2011;10:123-130.

Corti M, Patten C, Triggs W. Repetitive transcranial magnetic stimulation of motor cortex after stroke: a focused review. Am J Phys Med Rehabil. 2012;91:254-270.

Dancause N, Barbay S, Frost SB, Plautz EJ, Chen D, Zoubina EV, Stowe AM, Nudo RJ. Extensive cortical rewiring after brain injury. J Neurosci. 2005;25:10167-10179.

Ding Y, Li J, Clark J, Diaz FG, Rafols JA. Synaptic plasticity in thalamic nuclei enhanced by motor skill training in rat with transient middle cerebral artery occlusion. Neurol Res. 2003;25:189-194.

Dromerick AW, Lang CE, Birkenmeier RL, Wagner JM, Miller JP, Videen TO, Powers WJ, Wolf SL, Edwards DF. Very Early Constraint-Induced Movement during Stroke Rehabilitation (VECTORS): A single-center RCT. Neurology. 2009;73:195-201.

Ellis MD, Sukal-Moulton T, Dewald JP. Progressive shoulder abduction loading is a crucial element of arm rehabilitation in chronic stroke. Neurorehabil Neural Repair. 2009;23:862-869.

Hidler J, Nichols D, Pelliccio M, Brady K, Campbell DD, Kahn JH, Hornby TG. Multicenter randomized clinical trial evaluating the effectiveness of the Lokomat in subacute stroke. Neurorehabil Neural Repair. 2009;23:5-13.

Khedr EM, Ahmed MA, Fathy N, Rothwell JC. Therapeutic trial of repetitive transcranial magnetic stimulation after acute ischemic stroke. Neurology. 2005;65:466-468.

Kozlowski DA, James DC, Schallert T. Use-dependent exaggeration of neuronal injury after unilateral sensorimotor cortex lesions. J Neurosci. 1996;16:4776-4786.

Kwakkel G, van Peppen R, Wagenaar RC, Wood Dauphinee S, Richards C, Ashburn A, Miller K, Lincoln N, Partridge C, Wellwood I, Langhorne P. Effects of augmented exercise therapy time after stroke: a metaanalysis. Stroke. 2004;35:2529-2539.

Kwakkel G, Wagenaar RC, Twisk JW, Lankhorst GJ, Koetsier JC. Intensity of leg and arm training after primary middle-cerebral-artery stroke: a randomised trial. Lancet. 1999;354:191-196.

Lo AC, Guarino PD, Richards LG, Haselkorn JK, Wittenberg GF, Federman DG, Ringer RJ, Wagner TH, Krebs HI, Volpe BT, Bever CT, Bravata DM, Duncan PW, Corn BH, Maffucci AD, Nadeau SE, Conroy SS, 
Powell JM, Huang GD, Peduzzi P. Robot-Assisted Therapy for Long-Term Upper-Limb Impairment after Stroke. N Engl J Med. 2010;362:1772-1783.

Luft AR, McCombe-Waller S, Whitall J, Forrester LW, Macko R, Sorkin JD, Schulz JB, Goldberg AP, Hanley DF. Repetitive bilateral arm training and motor cortex activation in chronic stroke: a randomized controlled trial. JAMA. 2004;292:1853-1861.

Mizutani K, Sonoda S, Hayashi N, Takasaki A, Beppu H, Saitoh E, Shimpo K. Analysis of protein expression profile in the cerebellum of cerebral infarction rats after treadmill training. Am $\mathrm{J}$ Phys Med Rehabil. 2010;89:107-114.

Monti A, Cogiamanian F, Marceglia S, Ferrucci R, Mameli F, Mrakic-Sposta S, Vergari M, Zago S, Priori A. Improved naming after transcranial direct current stimulation in aphasia. J Neurol Neurosurg Psychiatry. 2008;79:451-453.

Nudo RJ, Milliken GW. Reorganization of movement representations in primary motor cortex following focal ischemic infarcts in adult squirrel monkeys. J Neurophysiol. 1996;75:2144-2149.

Nudo RJ, Wise BM, SiFuentes F, Milliken GW. Neural substrates for the effects of rehabilitative training on motor recovery after ischemic infarct. Science. 1996;272:1791-1794.

Ramanathan D, Conner JM, Tuszynski MH. A form of motor cortical plasticity that correlates with recovery of function after brain injury. Proc Natl Acad Sci U S A. 2006;103:11370-11375.

Riley JD, Le V, Der-Yeghiaian L, See J, Newton JM, Ward NS, Cramer SC. Anatomy of stroke injury predicts gains from therapy. Stroke. 2011;42:421-426.

Stinear CM, Barber PA, Smale PR, Coxon JP, Fleming MK, Byblow WD. Functional potential in chronic stroke patients depends on corticospinal tract integrity. Brain. 2007;130:170-180.

Stuart M, Benvenuti F, Macko R, Taviani A, Segenni L, Mayer F, Sorkin JD, Stanhope SJ, Macellari V, Weinrich M. Community-based adaptive physical activity program for chronic stroke: feasibility, safety, and efficacy of the Empoli model. Neurorehabil Neural Repair. 2009;23:726-734.

Teasell R, Bitensky J, Salter K, Bayona NA. The role of timing and intensity of rehabilitation therapies. Top Stroke Rehabil. 2005;12:46-57.

Wang RY, Tseng HY, Liao KK, Wang CJ, Lai KL, Yang YR. rTMS combined with task-oriented training to improve symmetry of interhemispheric corticomotor excitability and gait performance after stroke: a randomized trial. Neurorehabil Neural Repair. 2012;26:222-230.

Ward NS, Brown MM, Thompson AJ, Frackowiak RS. Neural correlates of motor recovery after stroke: a longitudinal fMRI study. Brain. 2003;126:2476-2496.

Werhahn KJ, Conforto AB, Kadom N, Hallett M, Cohen LG. Contribution of the ipsilateral motor cortex to recovery after chronic stroke. Ann Neurol. 2003;54:464-472.

Whitall J, Waller McCombe S, Sorkin JD, Forrester LW, Macko RF, Hanley DF, Goldberg AP, Luft AR. Bilateral and unilateral arm training improve motor function through differing neuroplastic mechanisms: a single-blinded randomized controlled trial. Neurorehabil Neural Repair. 2011;25:118-129.

Wolf SL, Winstein CJ, Miller JP, Taub E, Uswatte G, Morris D, Giuliani C, Light KE, Nichols-Larsen D, EXCITE Investigators. Effect of constraint-induced movement therapy on upper extremity function 3 to 9 months after stroke: the EXCITE randomized clinical trial. JAMA. 2006;296:2095-2104.

Wu D, Qian L, Zorowitz RD, Zhang L, Qu Y, Yuan Y. Effects on Decreasing Upper-Limb Poststroke Muscle Tone Using Transcranial Direct Current Stimulation: A Randomized Sham-Controlled Study. Arch Phys Med Rehabil. 2012.

You DS, Kim DY, Chun MH, Jung SE, Park SJ. Cathodal transcranial direct current stimulation of the right Wernicke's area improves comprehension in subacute stroke patients. Brain Lang. 2011;119:1-5. 